\title{
Nambu-Goldstone mechanism in real-time thermal field theory
}

\author{
Bang-Rong Zhou \\ Department of Physics, Graduate School at Beijing, University of Science and Technology of China \\ Academia Sinica, Beijing 100039, China* \\ and \\ The Abdus Salam International Centre for Theoretical Physics, P.O.Box 586, 34100 Trieste, Italy
}

\begin{abstract}
In a one-generation fermion condensate scheme of electroweak symmetry breaking, it is proved based on the Schwinger-Dyson equation in real-time thermal field theory in the fermion bubble diagram approximation that, at finite temperature $T$ below the symmetry restoration temperature $T_{c}$, a massive Higgs boson and three massless Nambu-Goldstone bosons could emerge from spontaneous breaking of the electroweak group $\mathrm{SU}_{L}(2) \times \mathrm{U}_{Y}(1) \rightarrow \mathrm{U}_{Q}(1)$ if the two fermion flavors in the one generation are mass degenerate, thus the Goldstone theorem is rigorously valid in this case. However, if the two fermion flavors have unequal masses, owing to "thermal fluctuation", the Goldstone theorem will be true only approximately for a very large momentum cutoff $\Lambda$ in the zero temperature fermion loop or for low energy scales. All possible pinch singularities are proved to cancel each other, as expected in a real-time thermal field theory.
\end{abstract}

14.80.Mz, 11.10.Wx, 11.30.Qc, 12.15.-y

\section{INTRODUCTION}

Spontaneous symmetry breaking at finite temperature has been investigated extensively [1-6]. However, most research by now has concentrated on the discussions of phase transition and critical temperature based on the effective potential approach at finite temperature, and relatively less work is reported about the theoretical exploration of the Nambu-Goldstone theorem [7] at finite temperature, especially in models of dynamical symmetry breaking such as the Nambu-Jona-Lasinio (NJL) model with four-fermion interactions [7]. Research into the Nambu-Goldstone mechanism at finite temperature could provide us a deeper understanding of the spontaneous breaking of a continuous symmetry at finite temperature and is certainly quite interesting. The key point of such research lies in verifying existence of Nambu-Goldstone bosons, i.e., determining the physical masses of the fermionic scalar and pseudoscalar bound states which appear as the products of spontaneous symmetry breaking. In a model of NJL form, the mass determination can be simply made by using Schwinger-Dyson equations, i.e., calculating directly the gap equation of fermion mass and the bound state propagators induced by the four-fermion interactions. On the other hand, it is possible that a scalar or pseudoscalar bound state is composed of fermions with different masses or is a combination of the scalar or pseudoscalar bilinears of these fermions [8-10]. Although a conventional effective potential approach is applicable to such models if one introduces axialiary scalar fields and replaces the four-fermion interactions by Yukawa couplings between axialiary scalar fields and fermion fields [6], it is unknown, by the introduction of axialiary scalar fields, whether one could include the effect of the mass diffrence of the constituent fermions of a bound state. However, we believe that the Schwinger-Dyson equation can surely do that. Therefore, to determine directly the masses of the bound states and to examine fully the effect of the fermion mass difference at finite temperature, we prefer the Schwinger-Dyson equation at finite temperature to a temperature effective potential. All the calculations will be conducted in the fermion bubble diagram approximation which amounts to the leading order of the $1 / \mathrm{N}$ expansion.

Since the determination of physical masses of the bound states is the key point of our research; it is certainly more convenient to take the real-time formalism of thermal field theory [4] than the imaginary-time formalism. In this way we will be able to avoid the cumbersome analytic continuation which is necessary in the latter formalism. However, again, because of the possible mass differences of the constituent fermions inside the bound states, the use of the real-time formalism will present a new question, i.e., whether this formalism consistent is with Nambu-Goldstone mechanism. In a simple model with $\mathrm{U}_{L}(N) \times \mathrm{U}_{R}(N)$-invariant four-fermion interactions, it has been proved that in the real-time formalism of thermal field theory the Nambu-Goldstone mechanism works indeed, but it is under the

*Permanent address. 
assumption that the fermions are of equal masses and equal chemical potentials [11]. In this paper, among other things, to examine further the stated consistency, we will consider a more realistic model - a one-generation fermion condensate scheme of electroweak symmetry breaking [9]. The extension to the many-generation case [10] is direct.

The paper is arranged as follows. In Sec. II we present the model, give its Lagrangian and derive the gap equation at finite temperature. In Secs. III, IV and V we will respectively calculate propagators of the scalar, the pseudoscalar, and the charged-scalar bound states at finite temperature by means of the Schwinger-Dyson equations satisfied by the fermionic four-point Green functions, determine the bound states' masses, and discuss the pinch singularity problem which is especially related to the real-time formalism of thermal field theory. Finally, in Sec. VI we come to our conclusions.

\section{ONE-GENERATION FERMION CONDENSATE MODEL AND GAP EQUATION}

In this model of electroweak symmetry breaking, we have one generation of $Q$ fermions forming a $\mathrm{SU}_{L}(2) \times \mathrm{U}_{Y}(1)$ doublet $(U, D)$ and are assigned in the representation $R$ of the color group $\mathrm{SU}_{c}(3)$ with the dimension $d_{Q}(R)$. The symmetry breaking is induced by the effective four-fermion interactions among the $Q$ fermions below some high momentum scale $\Lambda$ which, in real-time thermal field theory, are described by the Lagrangian $[4,9,10]$

$$
\mathcal{L}_{4 F}=\mathcal{L}_{4 F}^{S}+\mathcal{L}_{4 F}^{P}+\mathcal{L}_{4 F}^{C}
$$

The neutral scalar couplings

$$
\begin{gathered}
\mathcal{L}_{4 F}^{S}=\frac{1}{4} \sum_{a=1}^{2} \sum_{Q, Q^{\prime}}(-1)^{a+1} g_{Q^{\prime} Q}\left(\bar{Q}^{\prime} Q^{\prime}\right)^{(a)}(\bar{Q} Q)^{(a)} \\
g_{Q^{\prime} Q}=g_{Q^{\prime} Q^{\prime}}^{1 / 2} g_{Q Q}^{1 / 2}, \quad Q, Q^{\prime}=U, D
\end{gathered}
$$

where $a=1$ denotes physical fields and $a=2$ ghost fields. The physical fields and the ghost fields interact only through the propagators. The neutral pseudoscalar couplings

$$
\begin{gathered}
\left.\mathcal{L}_{4 F}^{P}=\frac{1}{4} \sum_{a=1}^{2} \sum_{Q, Q^{\prime}}(-1)^{a+1} g_{Q^{\prime} Q}^{\prime}\left(\bar{Q}^{\prime} i \gamma_{5} Q^{\prime}\right)^{(a)} \bar{Q} i \gamma_{5} Q\right)^{(a)} \\
g_{Q^{\prime} Q}^{\prime}=(-1)^{I_{Q^{\prime}}^{3}-I_{Q}^{3}} g_{Q^{\prime} Q}, \quad Q, Q^{\prime}=U, D
\end{gathered}
$$

and $I_{Q}^{3}$ denotes the third component of the weak isospin of the $Q$ fermions. The charged scalar couplings

$$
\begin{gathered}
\mathcal{L}_{4 F}^{C}=\frac{G}{2} \sum_{a=1}^{2}(-1)^{a+1}\left(\bar{D} \Gamma^{+} U\right)^{(a)}\left(\bar{U} \Gamma^{-} D\right)^{(a)}, \\
\Gamma^{ \pm}=\frac{1}{\sqrt{2}}\left[\cos \varphi-\sin \varphi \pm(\cos \varphi+\sin \varphi) \gamma_{5}\right], \\
G=g_{U U}+g_{D D}, \quad \cos ^{2} \varphi=g_{U U} / G, \quad \sin ^{2} \varphi=g_{D D} / G .
\end{gathered}
$$

We indicate that Lagrangian (2.1) is the real-time thermal field theory version of the following zero-temperature four-fermion Lagrangian for $n=1$ [10]:

$$
\mathcal{L}_{4 F}^{0}=\frac{G}{4}\left[\left(\phi_{S}^{0}\right)^{2}+\left(\phi_{P}^{0}\right)^{2}+2 \phi^{+} \phi^{-}\right],
$$

where 


$$
\begin{gathered}
\phi_{S}^{0}=\cos \varphi(\bar{U} U)^{(1)}+\sin \varphi(\bar{D} D)^{(1)}, \\
\phi_{P}^{0}=\cos \varphi\left(\bar{U} i \gamma_{5} U\right)^{(1)}-\sin \varphi\left(\bar{D} i \gamma_{5} D\right)^{(1)}, \\
\phi^{-}=\left(\bar{U} \Gamma^{-} D\right)^{(1)}, \quad \phi^{+}=\left(\bar{D} \Gamma^{+} U\right)^{(1)}
\end{gathered}
$$

are, respectively, the configurations of the physical neutral scalar, neutral pseudoscalar, and charged scalar bound states which are expressed by physical fermion fields with $a=1$. In zero-temperature field theory, one assumes that $\mathcal{L}_{4 F}^{S}$ will lead to the vacuum expectation value $\sum_{Q=U, D} g_{Q Q}\left\langle(\bar{Q} Q)^{(1)}\right\rangle \neq 0$ and this will induce spontaneous breaking of electroweak group. At finite temperature $T$ and in the real-time formalism of thermal field theory, we will assume the thermal expectation value $\sum_{Q=U, D} g_{Q Q}\left\langle(\bar{Q} Q)^{(1)}\right\rangle_{T} \neq 0$, where only the physical fields $(a=1)$ are considered [12]. We thus obtain the mass of the $\mathrm{Q}$ fermion,

$$
m_{Q}(T, \mu) \equiv m_{Q}=-\frac{1}{2} g_{Q Q}^{1 / 2} \sum_{Q^{\prime}=U, D} g_{Q^{\prime} Q^{\prime}}^{1 / 2}\left\langle\left(\bar{Q}^{\prime} Q^{\prime}\right)^{(1)}\right\rangle_{T},
$$

which will lead to the relation

$$
m_{Q} / g_{Q Q}^{1 / 2}=m_{Q^{\prime}} / g_{Q^{\prime} Q^{\prime}}^{1 / 2}
$$

and the gap equation at finite temperature $T$,

$$
1=\sum_{Q=U, D} g_{Q Q} I_{Q}
$$

with

$$
\begin{aligned}
I_{Q} & =-\frac{1}{2 m_{Q}}\left\langle(\bar{Q} Q)^{(1)}\right\rangle_{T}=\frac{d_{Q}(R)}{2 m_{Q}} \int \frac{d^{4} l}{(2 \pi)^{4}} \operatorname{tr}\left[i S^{11}\left(l, m_{Q}\right)\right] \\
& =2 d_{Q}(R) \int \frac{d^{4} l}{(2 \pi)^{4}}\left[\frac{i}{l^{2}-m_{Q}^{2}+i \varepsilon}-2 \pi \delta\left(l^{2}-m_{Q}^{2}\right) \sin ^{2} \theta\left(l^{0}, \mu_{Q}\right)\right],
\end{aligned}
$$

where we have used the thermal propagator of fermion in the matrix form

$$
\begin{aligned}
\left(\begin{array}{cc}
i S^{11}\left(l, m_{Q}\right), & i S^{12}\left(l, m_{Q}\right) \\
i S^{21}\left(l, m_{Q}\right), & i S^{22}\left(l, m_{Q}\right)
\end{array}\right)= & \left(\begin{array}{cc}
i /\left(y-m_{Q}+i \varepsilon\right), & 0 \\
0, & -i /\left(y-m_{Q}-i \varepsilon\right)
\end{array}\right) \\
& -2 \pi\left(y+m_{Q}\right) \delta\left(l^{2}-m_{Q}^{2}\right)\left(\begin{array}{cc}
\sin ^{2} \theta\left(l^{0}, \mu_{Q}\right), & \frac{1}{2} e^{\beta \mu_{Q} / 2} \sin 2 \theta\left(l^{0}, \mu_{Q}\right) \\
-\frac{1}{2} e^{-\beta \mu_{Q} / 2} \sin 2 \theta\left(l^{0}, \mu_{Q}\right), & \sin ^{2} \theta\left(l^{0}, \mu_{Q}\right)
\end{array}\right),
\end{aligned}
$$

with the chemical potential $\mu_{Q}$ of the $Q$ fermion and the denotations

$$
\sin ^{2} \theta\left(l^{0}, \mu_{Q}\right)=\frac{\theta\left(l^{0}\right)}{\exp \left[\beta\left(l^{0}-\mu_{Q}\right)\right]+1}+\frac{\theta\left(-l^{0}\right)}{\exp \left[\beta\left(-l^{0}+\mu_{Q}\right)\right]+1}
$$

and $\beta=1 / T$. The gap equation (2.9) could be satisfied merely at lower temperature $T$ than $T_{c}$, where $T_{c}$ is the critical temperature above which Eq. (2.9) is no longer valid and thus electroweak symmetry restoration is implied $[12,13]$. In view of this, in the following discussion we will assume $T<T_{c}$ so that the gap equation (2.9) can always be used.

\section{SCALAR BOUND STATE MODE}

The propagators for fermionic bound states correspond to the four-point Green functions of the fermions. Since there exist two types of four-fermion interaction vertices $(a=1,2)$ in real-time thermal field theory, the four-point Green functions will also be a matrix with the row and the column denoted by the index $a$. The four-point functions 
for the transition from $(\bar{Q} Q)^{(a)}$ to $\left(\bar{Q}^{\prime} Q^{\prime}\right)^{(b)}$ can be denoted by $\Gamma_{S}^{Q^{\prime} b Q a}(p)$; then, from Eq. $(2.2)$, they will obey the following linear algebraic equations [10]:

$$
\sum_{c} \sum_{Q^{\prime \prime}} \Gamma_{S}^{Q^{\prime} b Q^{\prime \prime} c}(p)\left[\delta_{Q^{\prime \prime} Q} \delta^{c a}-N_{Q^{\prime \prime}}^{c a}(p) g_{Q^{\prime \prime} Q}(-1)^{a+1}\right]=\frac{i}{2} g_{Q^{\prime} Q} \delta^{b a}(-1)^{a+1}, \quad Q^{\prime}, Q=U, D, \quad b, a=1,2,
$$

where $p$ is the four-momentum of the bound state, and $-2 i N_{Q}^{c a}$ represents the contribution of the $Q$-fermion loop with an $a$-type and a $c$-type scalar coupling vertex [Eq.(2.2)], i.e.,

$$
N_{Q}^{c a}(p)=-\frac{i}{2} d_{Q}(R) \int \frac{d^{4} l}{(2 \pi)^{4}} \operatorname{tr}\left[i S^{c a}\left(l, m_{Q}\right) i S^{a c}\left(l+p, m_{Q}\right)\right] .
$$

Equations (3.1) have the solutions

$$
\begin{aligned}
\Gamma_{S}^{Q^{\prime} b Q a}(p)= & \frac{i}{2 \Delta_{S}(p)} g_{Q^{\prime} Q}\left\{\left(\delta^{b 1}\left[1+\sum_{Q} g_{Q Q} N_{Q}^{22}(p)\right]-\delta^{b 2} \sum_{Q} g_{Q Q} N_{Q}^{21}(p)\right) \delta^{a 1}\right. \\
& \left.-\left(\delta^{b 2}\left[1-\sum_{Q} g_{Q Q} N_{Q}^{11}(p)\right]+\delta^{b 1} \sum_{Q} g_{Q Q} N_{Q}^{12}(p)\right) \delta^{a 2}\right\}, \quad Q^{\prime}, Q=U, D, \quad b, a=1,2,
\end{aligned}
$$

where the coefficient determinant of Eqs. (3.1),

$$
\Delta_{S}(p)=\left[1-\sum_{Q} g_{Q Q} N_{Q}^{11}(p)\right]\left[1+\sum_{Q} g_{Q Q} N_{Q}^{22}(p)\right]+\left[\sum_{Q} g_{Q Q} N_{Q}^{12}(p)\right]\left[\sum_{Q} g_{Q Q} N_{Q}^{21}(p)\right] .
$$

The propagator for the physical scalar bound state $\phi_{S}^{0}$ expressed in Eq. (2.6) is

$$
\begin{aligned}
\Gamma^{\phi_{S}^{0}}(p) & =\cos ^{2} \varphi \Gamma_{S}^{U 1 U 1}(p)+\sin ^{2} \varphi \Gamma_{S}^{D 1 D 1}(p)+\sin \varphi \cos \varphi\left[\Gamma_{S}^{D 1 U 1}(p)+\Gamma_{S}^{U 1 D 1}(p)\right] \\
& =i G\left[1+\sum_{Q} g_{Q Q} N_{Q}^{22}(p)\right] / 2 \Delta_{S}(p) .
\end{aligned}
$$

The problem is reduced to the calculation of $N_{Q}^{a b}(p)$. By using the formula

$$
\frac{1}{X+i \varepsilon}=\frac{X}{X^{2}+\varepsilon^{2}}-i \pi \delta(X)
$$

and through direct but rather lengthy derivation we obtain

$$
\begin{aligned}
N_{Q}^{11}(p) & =I_{Q}+\frac{1}{2}\left(p^{2}-4 m_{Q}^{2}+i \varepsilon\right)\left[K_{Q}(p)+H_{Q}(p)-i S_{Q}(p)\right] \\
& =-\left[N_{Q}^{22}(p)\right]^{*}, \\
N_{Q}^{12}(p) & =N_{Q}^{21}(p)=-\frac{i}{2}\left(p^{2}-4 m_{Q}^{2}\right) R_{Q}(p),
\end{aligned}
$$

where

$$
\begin{aligned}
K_{Q}(p) & =-2 d_{Q}(R) \int \frac{i d^{4} l}{(2 \pi)^{4}} \frac{1}{\left(l^{2}-m_{Q}^{2}+i \varepsilon\right)\left[(l+p)^{2}-m_{Q}^{2}+i \varepsilon\right]} \\
& =\frac{d_{Q}(R)}{8 \pi^{2}} \int_{0}^{1} d x\left(\ln \frac{\Lambda^{2}+M_{Q}^{2}}{M_{Q}^{2}}-\frac{\Lambda^{2}}{\Lambda^{2}+M_{Q}^{2}}\right), \quad M_{Q}^{2}=m_{Q}^{2}-p^{2} x(1-x),
\end{aligned}
$$

with the four-dimension Euclidean momentum cutoff $\Lambda$, 


$$
\begin{aligned}
& H_{Q}(p)= 4 \pi d_{Q}(R) \int \frac{d^{4} l}{(2 \pi)^{4}}\left\{\frac{(l+p)^{2}-m_{Q}^{2}}{\left[(l+p)^{2}-m_{Q}^{2}\right]^{2}+\varepsilon^{2}}+(p \rightarrow-p)\right\} \delta\left(l^{2}-m_{Q}^{2}\right) \sin ^{2} \theta\left(l^{0}, \mu_{Q}\right) \\
&= \frac{1}{16 \pi^{2}|\vec{p}|} \int_{0}^{\infty} \frac{d|\vec{l} \| \vec{l}|}{\omega_{Q l}}\left[\ln \frac{\left(p^{2}-2 \omega_{Q l} p^{0}+2|\vec{l} \| \vec{p}|\right)^{2}+\varepsilon^{2}}{\left(p^{2}-2 \omega_{Q l} p^{0}+2|\vec{l} \| \vec{p}|\right)^{2}+\varepsilon^{2}}+\left(p^{0} \rightarrow-p^{0}\right)\right] \\
& {\left[\frac{1}{\exp \left[\beta\left(\omega_{Q l}-\mu_{Q}\right)\right]+1}+\frac{1}{\exp \left[\beta\left(\omega_{Q l}+\mu_{Q}\right)\right]+1}\right], \omega_{Q l}=\sqrt{\vec{l}^{2}+m_{Q}^{2}} } \\
& S_{Q}(p)=4 \pi^{2} d_{Q}(R) \int \frac{d^{4} l}{(2 \pi)^{4}} \delta\left(l^{2}-m_{Q}^{2}\right) \delta\left[(l+p)^{2}-m_{Q}^{2}\right]\left[\sin ^{2} \theta\left(l^{0}+p^{0}, \mu_{Q}\right) \cos ^{2} \theta\left(l^{0}, \mu_{Q}\right)+\cos ^{2} \theta\left(l^{0}+p^{0}, \mu_{Q}\right) \sin ^{2} \theta\left(l^{0}, \mu_{Q}\right)\right]
\end{aligned}
$$

and

$$
R_{Q}(p)=2 \pi^{2} d_{Q}(R) \int \frac{d^{4} l}{(2 \pi)^{4}} \delta\left(l^{2}-m_{Q}^{2}\right) \delta\left[(l+p)^{2}-m_{Q}^{2}\right] \sin 2 \theta\left(l^{0}, \mu_{Q}\right) \sin 2 \theta\left(l^{0}+p^{0}, \mu_{Q}\right) .
$$

In Eq.(3.9) the limit $\varepsilon \rightarrow 0$ will be taken only after the total calculations are completed. Substituting Eq. (3.7) into Eq. (3.5) and using the gap equation (2.9) and the relation

$$
g_{Q Q} / G=m_{Q}^{2} / \sum_{Q} m_{Q}^{2}
$$

derived from Eq. (2.8), we obtain

$$
\begin{aligned}
\Gamma^{\phi_{S}^{0}}(p)=-i \sum_{Q} m_{Q}^{2} / & \left\{\sum_{Q}\left(p^{2}-4 m_{Q}^{2}+i \varepsilon\right) m_{Q}^{2}\left[K_{Q}(p)+H_{Q}(p)-i S_{Q}(p)\right]\right. \\
& \left.-\frac{\left[\sum_{Q}\left(p^{2}-4 m^{2}\right) m_{Q}^{2} R_{Q}(p)\right]^{2}}{\sum_{Q}\left(p^{2}-4 m^{2}-i \varepsilon\right) m_{Q}^{2}\left[K_{Q}(p)+H_{Q}(p)+i S_{Q}(p)\right]}\right\} .
\end{aligned}
$$

The mass squared $m_{\phi_{S}^{0}}^{2}$ is determined by the pole's position of $\Gamma^{\phi_{S}^{0}}(p)$, i.e., by the equation

$$
\left\{\sum_{Q}\left(p^{2}-4 m_{Q}^{2}\right) m_{Q}^{2}\left[K_{Q}(p)+H_{Q}(p)\right]\right\}^{2}+\left\{\sum_{Q}\left(p^{2}-4 m_{Q}^{2}\right) m_{Q}^{2} S_{Q}(p)\right\}^{2}=\left\{\sum_{Q}\left(p^{2}-4 m_{Q}^{2}\right) m_{Q}^{2} R_{Q}(p)\right\}^{2} .
$$

We notice that when $\Lambda^{2}>>M_{Q}^{2}$,

$$
\begin{aligned}
K_{Q}(p)= & \frac{d_{Q}(R)}{8 \pi^{2}}\left(\ln \frac{\Lambda^{2}}{m_{Q}^{2}}+1\right) \\
& -\frac{d_{Q}(R)}{8 \pi^{2}}\left\{\begin{array}{lll}
\sqrt{\lambda_{Q}-1} \arctan \frac{1}{\sqrt{\lambda_{Q}-1}} & \text { if } & \lambda_{Q}>1, \\
\sqrt{1-\lambda_{Q}} \ln \frac{1+\sqrt{1-\lambda_{Q}}}{1-\sqrt{1-\lambda_{Q}}}, & \text { if } & \lambda_{Q}<1,
\end{array}, \lambda_{Q}=4 m_{Q}^{2} / p^{2} .\right.
\end{aligned}
$$

Hence $K_{Q}(p)$ is real and positive definite. The same conclusion is true with $H_{Q}(p)$ and $S_{Q}(p)$ since the integrands in Eqs. (3.9) and (3.10) are real and positive, but not applicable to $R_{Q}(p)$. In addition, we have the inequalities

$$
S_{Q}(p) \pm R_{Q}(p)=4 \pi^{2} d_{Q}(R) \int \frac{d^{4} l}{(2 \pi)^{4}} \delta\left(l^{2}-m_{Q}^{2}\right) \delta\left[(l+p)^{2}-m_{Q}^{2}\right] \sin ^{2}\left[\theta\left(l^{0}+p^{0}, \mu_{Q}\right) \pm \theta\left(l^{0}, \mu_{Q}\right)\right] \geq 0 .
$$

Let 


$$
\begin{gathered}
\sum_{Q} m_{Q}^{2} K_{Q}(p)=k, \quad \sum_{Q} m_{Q}^{2} H_{Q}(p)=h, \quad \sum_{Q} m_{Q}^{2} S_{Q}(p)=s, \quad \sum_{Q} m_{Q}^{2} R_{Q}(p)=r, \\
\sum_{Q} m_{Q}^{4} K_{Q}(p)=\tilde{k}, \quad \sum_{Q} m_{Q}^{4} H_{Q}(p)=\tilde{h}, \quad \sum_{Q} m_{Q}^{4} S_{Q}(p)=\tilde{s}, \quad \sum_{Q} m_{Q}^{4} R_{Q}(p)=\tilde{r} .
\end{gathered}
$$

then Eq. (3.14) can be rewritten as

$$
\left[(k+h) p^{2}-4(\tilde{k}+\tilde{h})\right]^{2}+\left(s p^{2}-4 \tilde{s}\right)^{2}=\left(r p^{2}-4 \tilde{r}\right)^{2} .
$$

It has the solutions

$$
\begin{aligned}
& p^{2}= 4 \frac{[(k+h)(\tilde{k}+\tilde{h})+s \tilde{s}-r \tilde{r}]}{(k+h)^{2}+s^{2}-r^{2}}\{1 \pm A\} \\
& A=\left\{1-\frac{\left[(k+h)^{2}+s^{2}-r^{2}\right]\left[(\tilde{k}+\tilde{h})^{2}+\tilde{s}^{2}-\tilde{r}^{2}\right]}{[(k+h)(\tilde{k}+\tilde{h})+s \tilde{s}-r \tilde{r}]^{2}}\right\}^{1 / 2}
\end{aligned}
$$

Equation (3.19) shows that we could obtain two different $m_{\phi_{S}^{0}}^{2}$. However, we indicate that, first of all, in the special case where only single-flavor $Q$ fermions exist (e.g., in the top-quark condensate scheme [8]) or all the $m_{Q}$ are equal (mass degenerate), the $A$ will be identical to zero and we still have only a single $m_{\phi_{S}^{0}}$. Next, in general case, if the momentum cut-off $\Lambda$ is large enough, then, considering Eq. (3.16), we always have

$$
(k+h)^{2}>>s^{2}-r^{2}>0, \quad(\tilde{k}+\tilde{h})^{2}>>\tilde{s}^{2}-\tilde{r}^{2}>0, \quad(k+h)(\tilde{k}+\tilde{h})>>s \tilde{s}-r \tilde{r},
$$

and thus $A \approx 0$. In fact, $A \neq 0$ can be explained as thermal fluctuation of the squared mass of $\phi_{S}^{0}$. In any way, we may consider that physically the mass of $\phi_{S}^{0}$ is determined by the equation

$$
\begin{aligned}
m_{\phi_{S}^{0}}^{2}=p^{2} & \left.\simeq \frac{4}{(k+h)^{2}+s^{2}-r^{2}}[(k+h)(\tilde{k}+\tilde{h})+s \tilde{s}-r \tilde{r}]\right|_{p^{2}=m_{\phi_{S}^{2}}^{2}} \\
& =\left.\frac{4(k+h)(\tilde{k}+\tilde{h})+2[(s+r)(\tilde{s}-\tilde{r})+(\tilde{s}+\tilde{r})(s-r)]}{(k+h)^{2}+s^{2}-r^{2}}\right|_{p^{2}=m_{\phi_{S}^{0}}^{2}} .
\end{aligned}
$$

Based on Eq. (3.16) we have $s-r \geq 0$ and $\tilde{s}-\tilde{r} \geq 0$; thus it is deduced from Eq. (3.20) that

$$
2\left(m_{Q}\right)_{\min } \leq m_{\phi_{S}^{0}} \leq 2\left(m_{Q}\right)_{\max }
$$

where $\left(m_{Q}\right)_{\min }$ and $\left(m_{Q}\right)_{\max }$ are, respectively, the minimal and the maximal mass among the $Q$ fermions. The limitation (3.21) is formally the same as the one at zero temperature [10], but it should be understood that $m_{Q} \equiv$ $m_{Q}\left(T, \mu_{Q}\right)$ is now the $Q$-fermion mass at $T \neq 0$.

When $p \rightarrow 0$, by Eq. (3.9), $H_{Q}(p)=0$ and by Eqs. (3.10) and (3.11), $S_{Q}(p)$ and $R_{Q}(p)$ contain the pinch singularities. However, we obtain from Eq. (3.16) that

$$
\left.\left[S_{Q}(p)-R_{Q}(p)\right]\right|_{p \rightarrow 0}=0 \text { or }\left.(\tilde{s}-\tilde{r})\right|_{p \rightarrow 0}=0
$$

and this will make the pinch singularities contained in the denominator of $\Gamma^{\phi_{S}^{0}}(p)$ cancel each other. This result is just expected in real-time thermal field theory.

In addition, we also indicate that it is easy to verify by Eq. (3.3) that, similar to the zero-temperature case, for the orthogonal combination to $\phi_{S}^{0}$,

$$
\tilde{\phi}_{S}^{0}=-\sin \varphi(\bar{U} U)^{(1)}+\cos \varphi(\bar{D} D)^{(1)},
$$

its propagator $\Gamma^{\tilde{\phi}_{S}^{0}}(p)=0$, i.e., the configuration $\tilde{\phi}_{S}^{0}$, does not exist. We only have the single neutral scalar bound state $\phi_{S}^{0}$ left. 


\section{PSEUDOSCALAR BOUND STATE MODE}

The calculation of the propagator for pseudoscalar bound state is similar to the one for scalar bound state. The four-point function for transition from $\left(\bar{Q} i \gamma_{5} Q\right)^{(a)}$ to $\left(\bar{Q}^{\prime} i \gamma_{5} Q^{\prime}\right)^{(b)}$ is denoted by $\Gamma_{P}^{Q^{\prime} b Q a}$; then from Eq. (2.3) they will obey the linear algebraic equations [10]

$$
\sum_{c} \sum_{Q "} \Gamma_{P}^{Q^{\prime} b Q^{\prime \prime} c}(p)\left[\delta_{Q "}{ }^{c a}-N_{Q "}^{c a}(p) g_{Q " Q^{\prime}}^{\prime}(-1)^{a+1}\right]=\frac{i}{2} g_{Q^{\prime} Q^{\prime}}^{\delta^{b a}}(-1)^{a+1}, \quad Q^{\prime}, Q=U, D, \quad b, a=1,2 .
$$

We have used the denotation

$$
N_{Q 5}^{c a}(p)=-\frac{i}{2} d_{Q}(R) \int \frac{d^{4} l}{(2 \pi)^{4}} \operatorname{tr}\left[i \gamma_{5} i S^{c a}\left(l, m_{Q}\right) i \gamma_{5} i S^{a c}\left(l+p, m_{Q}\right)\right],
$$

and $-2 i N_{Q 5}^{c a}(p)$ represents the contribution of the $Q$-fermion loop with an $a$-type and a $c$-type pseudoscalar coupling vertex [Eq. (2.3)]. By comparing Eqs. (3.1) with Eqs. (4.1), it is easy to find that the solutions of Eqs. (4.1) can be obtained from the solutions (3.3) by the substitutions

$$
N_{Q}^{c a}(p) \rightarrow N_{Q 5}^{c a}(p), \quad g_{Q^{\prime} Q} \rightarrow g_{Q^{\prime} Q}^{\prime}
$$

Thus

$$
\begin{aligned}
\Gamma_{P}^{Q^{\prime} b Q a}(p)= & \frac{i}{2 \Delta_{S}(p)} g_{Q^{\prime} Q}^{\prime}\left\{\left(\delta^{b 1}\left[1+\sum_{Q} g_{Q Q} N_{Q 5}^{22}(p)\right]-\delta^{b 2} \sum_{Q} g_{Q Q} N_{Q 5}^{21}(p)\right) \delta^{a 1}\right. \\
& \left.-\left(\delta^{b 2}\left[1-\sum_{Q} g_{Q Q} N_{Q 5}^{11}(p)\right]+\delta^{b 1} \sum_{Q} g_{Q Q} N_{Q 5}^{12}(p)\right) \delta^{a 2}\right\}, \quad Q^{\prime}, Q=U, D, \quad b, a=1,2,
\end{aligned}
$$

where

$$
\Delta_{P}(p)=\left[1-\sum_{Q} g_{Q Q} N_{Q 5}^{11}(p)\right]\left[1+\sum_{Q} g_{Q Q} N_{Q 5}^{22}(p)\right]+\left[\sum_{Q} g_{Q Q} N_{Q 5}^{12}(p)\right]\left[\sum_{Q} g_{Q Q} N_{Q 5}^{21}(p)\right]
$$

The propagator of the physical neutral pseudoscalar bound state $\phi_{P}^{0}$ defined in Eq. (2.6) is

$$
\begin{aligned}
\Gamma_{P}^{\phi_{P}^{0}}(p) & =\cos ^{2} \varphi \Gamma_{P}^{U 1 U 1}(p)+\sin ^{2} \varphi \Gamma_{P}^{D 1 D 1}(p)-\sin \varphi \cos \varphi\left[\Gamma_{P}^{D 1 U 1}(p)+\Gamma_{P}^{U 1 D 1}(p)\right] \\
& =i G\left[1+\sum_{Q} g_{Q Q} N_{Q 5}^{22}(p)\right] / 2 \Delta_{P}(p) .
\end{aligned}
$$

For the orthogonal combination $\tilde{\phi}_{P}^{0}$ to $\phi_{P}^{0}$, we have

$$
\Gamma^{\tilde{\phi}_{P}^{0}}(p)=0, \quad \tilde{\phi}_{P}^{0}=\sin \varphi\left(\bar{U} i \gamma_{5} U\right)^{(1)}+\cos \varphi\left(\bar{D} i \gamma_{5} D\right)^{(1)}
$$

hence, only the single neutral pseudoscalar bound state $\phi_{P}^{0}$ exists. The calculations of $N_{Q 5}^{a b}$ in Eq. (4.2) are similar to the ones of $N_{Q}^{a b}$ in Eq. (3.2) and the results can be obtained by the substitutions

$$
N_{Q 5}^{a b}(p)=\left.N_{Q}^{a b}(p)\right|_{p^{2}-4 m_{Q}^{2} \rightarrow p^{2}} .
$$

By means of the gap equation (2.9) and the relation (3.12) we obtain from Eqs. (4.5), (4.4), (4.7), and (3.7) that

$$
\begin{aligned}
\Gamma^{\phi_{P}^{0}}(p) & =-i \sum_{Q} m_{Q}^{2} /\left(p^{2}+i \varepsilon\right)\left\{\sum_{Q} m_{Q}^{2}\left[K_{Q}(p)+H_{Q}(p)-i S_{Q}(p)\right]-\frac{\left[\sum_{Q} m_{Q}^{2} R_{Q}(p)\right]^{2}}{\sum_{Q} m_{Q}^{2}\left[K_{Q}(p)+H_{Q}(p)+i S_{Q}(p)\right]}\right\} \\
& =-i \sum_{Q} m_{Q}^{2} /\left(p^{2}+i \varepsilon\right)\left(k+h-i s-\frac{r^{2}}{k+h+i s}\right) .
\end{aligned}
$$


We notice that when $p \rightarrow 0, h=0$ and $s=r$ based on Eq. (3.21); so the terms containing the pinch singularities in the denominator of Eq. (4.8) will cancel each other and $\Gamma^{\phi_{P}^{0}}(p)$ becomes finite. The expression (4.8) shows that $p^{2}=0$ is a single pole of the propagator $\Gamma_{P}^{\phi_{P}^{0}}(p)$ and thus $\phi_{P}^{0}$ is a massless neutral pseudoscalar composite particle.

It is interesting to indicate that when $p^{2}=0, S_{Q}(p)=R_{Q}(p)=0$ in Eqs. (3.10) and (3.11) since the constraints $l^{2}=m_{Q}^{2},(l+p)^{2}=m_{Q}^{2}$, and $p^{2}=0$ could not be submitted simultaneously and in addition, based on Eq. (3.9), also $H_{Q}(p)=0$; so we will have

$$
\Gamma^{\phi_{P}^{0}}(p)=-i \sum_{Q} m_{Q}^{2} /\left(p^{2}+i \varepsilon\right) k \quad \text { if } \quad p^{2} \rightarrow 0,
$$

which has the identical form to the propagator for the neutral pseudoscalar bound state at $T=0$ [10], except that the $Q$-fermion mass $m_{Q}(T=0)$ is replaced by $m_{Q}\left(T, \mu_{Q}\right)$. The result implies that the mass of $\phi_{P}^{0}$ is not affected by a finite temperature completely.

\section{CHARGED SCALAR BOUND STATE MODE}

For the calculation of the propagator for charged scalar bound state, a new feature is that the fermion loop is constituted by the propagators of the $U$ and $D$ fermions possibly with different masses. Denote the four-point function for the transition from $\left(\bar{U} \Gamma^{-} D\right)^{(a)}$ to $\left(\bar{D} \Gamma^{+} U\right)^{(b)}$ by $\Gamma_{\phi^{-}}^{b a}(p)$; then, based on Eq. (2.4), they will obey the linear algebraic equations

$$
\sum_{c=1,2} \Gamma_{\phi^{-}}^{b c}(p)\left[\delta^{c a}-L^{c a}(p) G(-1)^{a+1}\right]=i \frac{G}{2} \delta^{b a}(-1)^{(a+1)}, \quad b, a=1,2,
$$

where

$$
L^{c a}(p)=-\frac{i}{2} d_{Q}(R) \int \frac{d^{4} l}{(2 \pi)^{4}} \operatorname{tr}\left[\Gamma^{-} i S^{c a}\left(l, m_{U}\right) \Gamma^{+} i S^{a c}\left(l+p, m_{D}\right)\right]
$$

and $-2 i L^{c a}(p)$ represents the contribution of the fermion loop composed of $U$-fermion and $D$-fermion propagators with an $a$-type $\Gamma^{+}$coupling vertex and a $c$-type $\Gamma^{-}$coupling vertex. The solutions of Eqs. (5.1) are

$$
\Gamma_{\phi^{-}}^{b a}(p)=\frac{i G}{2 \Delta_{C}(p)}\left(\left\{\left[1+G L^{22}(p)\right] \delta^{b 1}-G L^{21}(p) \delta^{b 2}\right\} \delta^{a 1}-\left\{\left[1-G L^{11}(p)\right] \delta^{b 2}+G L^{12}(p) \delta^{b 1}\right\} \delta^{a 2}\right),
$$

where

$$
\Delta_{C}(p)=\left[1-G L^{11}(p)\right]\left[1+G L^{22}(p)\right]+G^{2} L^{12}(p) L^{21}(p) .
$$

The propagator for physical charged scalar bound state $\phi^{-}$is

$$
\Gamma^{\phi^{-}}(p) \equiv \Gamma_{\phi^{-}}^{11}(p)=i G / 2\left[1-G L^{11}(p)+G^{2} \frac{L^{12}(p) L^{21}(p)}{1+G L^{22}(p)}\right]
$$

and for the orthogonal combination $\tilde{\phi}^{-}$to $\phi^{-}$we have

$$
\Gamma^{\tilde{\phi}^{-}}(p)=0, \quad \tilde{\phi}^{-}=\frac{1}{\sqrt{2}} \bar{U}\left[\cos \varphi+\sin \varphi+(\cos \varphi-\sin \varphi) \gamma_{5}\right] D,
$$

since $\mathcal{L}_{4 F}^{C}$ contains no configuration $\tilde{\phi}^{-}$. It is noticed that $\phi^{-}$and its hermitian conjugate $\phi^{+}$have the same propagator and they become the only two charged scalar bound states. Direct calculations give

$$
\begin{aligned}
L^{11}(p) & =\frac{1}{G} \sum_{Q} g_{Q Q} I_{Q}+\frac{\left(p^{2}+i \varepsilon\right)}{2}\left[K_{U D}(p)+H_{U D}(p)-i S_{U D}(p)\right]+\frac{1}{2}\left[E_{U D}(p)+i \frac{\left(m_{U}^{2}-m_{D}^{2}\right)^{2}}{m_{U}^{2}+m_{D}^{2}} S_{U D}(p)\right] \\
& =-\left[L^{22}(p)\right]^{*}, \\
L^{12}(p) & =-\frac{i}{2}\left[p^{2}-\frac{\left(m_{U}^{2}-m_{D}^{2}\right)^{2}}{m_{U}^{2}+m_{D}^{2}}\right] R_{U D}(p) \exp \left[\beta\left(\mu_{U}-\mu_{D}\right) / 2\right], \\
L^{21}(p) & =-\frac{i}{2}\left[p^{2}-\frac{\left(m_{U}^{2}-m_{D}^{2}\right)^{2}}{m_{U}^{2}+m_{D}^{2}}\right] R_{U D}(p) \exp \left[-\beta\left(\mu_{U}-\mu_{D}\right) / 2\right],
\end{aligned}
$$


where

$$
\begin{gathered}
K_{U D}(p)=\frac{d_{Q}(R)}{4 \pi^{2}} \int_{0}^{1} d x \frac{m_{U}^{2}(1-x)+m_{D}^{2} x}{m_{U}^{2}+m_{D}^{2}}\left[\ln \frac{\Lambda^{2}+M_{U D}^{2}(p)}{M_{U D}^{2}(p)}-\frac{\Lambda^{2}}{\Lambda^{2}+M_{U D}^{2}(p)}\right] \\
M_{U D}^{2}(p)=m_{U}^{2}(1-x)+m_{D}^{2} x-p^{2} x(1-x) \\
H_{U D}(p)=4 \pi d_{Q}(R) \int \frac{d^{4} l}{(2 \pi)^{4}}\left\{\frac{(l+p)^{2}-m_{D}^{2}}{\left[(l+p)^{2}-m_{D}^{2}\right]^{2}+\varepsilon^{2}} \delta\left(l^{2}-m_{U}^{2}\right) \sin ^{2} \theta\left(l^{0}, \mu_{U}\right)+\left(p \rightarrow-p, m_{U} \leftrightarrow m_{D}, \mu_{U} \leftrightarrow \mu_{D}\right)\right\}, \\
E_{U D}(p)=4 \pi d_{Q}(R) \frac{m_{U}^{2}-m_{D}^{2}}{m_{U}^{2}+m_{D}^{2}} \int \frac{d^{4} l}{(2 \pi)^{4}} \\
\left\{\frac{\left[(l+p)^{2}-m_{U}^{2}\right]\left[(l+p)^{2}-m_{D}^{2}\right]}{\left[(l+p)^{2}-m_{D}^{2}\right]^{2}+\varepsilon^{2}} \delta\left(l^{2}-m_{U}^{2}\right) \sin ^{2} \theta\left(l^{0}, \mu_{U}\right)-\left(p \rightarrow-p, m_{U} \leftrightarrow m_{D}, \mu_{U} \leftrightarrow \mu_{D}\right)\right\} \\
S_{U D}(p)=4 \pi^{2} d_{Q}(R) \int \frac{d^{4} l}{(2 \pi)^{4}} \delta\left(l^{2}-m_{U}^{2}\right) \delta\left[(l+p)^{2}-m_{D}^{2}\right] \\
\left\{\sin ^{2} \theta\left(l^{0}, \mu_{U}\right) \cos ^{2} \theta\left(l^{0}+p^{0}, \mu_{D}\right)+\cos ^{2} \theta\left(l^{0}, \mu_{U}\right) \sin ^{2} \theta\left(l^{0}+p^{0}, \mu_{D}\right)\right\} \\
R_{U D}(p)=2 \pi^{2} d_{Q}(R) \int \frac{d^{4} l}{(2 \pi)^{4}} \delta\left(l^{2}-m_{U}^{2}\right) \delta\left[(l+p)^{2}-m_{D}^{2}\right] \sin _{2} \theta\left(l^{0}, \mu_{U}\right) \sin 2 \theta\left(l^{0}+p^{0}, \mu_{D}\right) .
\end{gathered}
$$

Considering the gap equation (2.6) we obtain from Eqs. (5.5) and (5.7) the propagator for $\phi^{-}$:

$$
\begin{aligned}
\Gamma^{\phi^{-}}(p)= & -i /\left\{\left(p^{2}+i \varepsilon\right)\left[K_{U D}(p)+H_{U D}(p)-i S_{U D}(p)\right]+E_{U D}(p)+i \bar{M}^{2} S_{U D}(p)\right. \\
& \left.-\frac{\left(p^{2}-\bar{M}^{2}\right)^{2} R_{U D}^{2}(p)}{\left(p^{2}-i \varepsilon\right)\left[K_{U D}(p)+H_{U D}(p)+i S_{U D}(p)\right]+E_{U D}(p)-i \bar{M}^{2} S_{U D}(p)}\right\}
\end{aligned}
$$

with

$$
\bar{M}^{2}=\left(m_{U}^{2}-m_{D}^{2}\right)^{2} /\left(m_{U}^{2}+m_{D}^{2}\right) .
$$

The mass of $\phi^{-}\left(\phi^{+}\right)$will be determined by the zero point of the denominator of $\Gamma^{\phi^{-}}(p)$. An interesting question is that under what conditions $p^{2} \rightarrow 0$ is the pole of $\Gamma^{\phi^{-}}(p)$ so that $\phi^{-}$and $\phi^{+}$would become massless bound states. Let us discuss this problem in two cases.

(1) $m_{U}=m_{D}=m_{Q}$. That is, the two fermion flavors in one generation are mass degenerate. In this case, we have $K_{U D}(p)=K_{Q}(p), H_{U D}(p)=H_{Q}(p), S_{U D}(p)=S_{Q}(p), R_{U D}(p)=R_{Q}(p), E_{U D}(p)=0$, and $\bar{M}^{2}=0$. Thus

$$
\Gamma^{\phi^{-}}(p)=-i /\left(p^{2}+i \varepsilon\right)\left[K_{Q}(p)+H_{Q}(p)-i S_{Q}(p)-\frac{R_{Q}^{2}(p)}{K_{Q}(p)+H_{Q}(p)+i S_{Q}(p)}\right],
$$

which has a form similar to the propagator (4.8) of pseudoscalar bound state except that now no sum of $Q$ with the weight $m_{Q}^{2}$ exists. Therefore, it follows from Eq. (5.14) that $p^{2}=0$ is the single pole of $\Gamma^{\phi^{-}}(p)$ and $\phi^{-}$and $\phi^{+}$will be exactly massless charged bound states - charged Nambu-Goldstone bosons. In addition, similar to the case of $\Gamma_{P}^{\phi_{P}^{0}}$, when $p \rightarrow 0$ the pinch singularities appearing in $S_{Q}(p)$ and $R_{Q}(p)$ also cancel each other.

(2) $m_{U} \neq m_{D}$. In this case, we notice that no pinch singularity could appear. This may also be seen from the expressions (5.11) and (5.12) of $S_{U D}(p)$ and $R_{U D}(p)$ in which $\delta\left(l^{2}-m_{U}^{2}\right) \delta\left[(l+p)^{2}-m_{D}^{2}\right]$ in the integrands will be equal to be zero if $p=0$ and $m_{U} \neq m_{D}$. Therefore, we could calculate the propagator for $\phi^{-}$on the condition that the ghost fields with $a=2$ are omitted completely and obtain 


$$
\begin{aligned}
\Gamma^{\phi^{-}}(p) & =i G / 2\left[1-G L^{11}(p)\right] \\
& =-i /\left\{\left(p^{2}+i \varepsilon\right)\left[K_{U D}(p)+H_{U D}(p)-i S_{U D}(p)\right]+E_{U D}(p)+i \bar{M}^{2} S_{U D}(p)\right\} .
\end{aligned}
$$

Equation (5.16) may be obtained approximately from Eq. (5.13) by assuming that the momentum cutoff $\Lambda$ in $K_{U D}(p)$ is large enough to neglect terms containing $R_{U D}^{2}(p)$. The pole of $\Gamma^{\phi^{-}}(p)$ is determined by the equation

$$
p^{2}=-\frac{E_{U D}(p)+i \bar{M}^{2} S_{U D}(p)}{K_{U D}(p)+H_{U D}(p)-i S_{U D}(p)} .
$$

Equation (5.17) shows that at finite temperature it is possible that the single pole of $\Gamma^{\phi^{-}}(p)$ is not at $p^{2}=0$ and thus the masses of $\phi^{-}$and $\phi^{+}$are not equal to zeros. However, it is seen from the right-hand side of Eq. (5.17) that as long as the momentum cutoff $\Lambda$ is large enough, the single pole of $\Gamma^{\phi^{-}}(p)$ could still be approximately at $p^{2}=0$. In particular, we notice that when $p^{2}=0$ and $p^{0}=|\vec{p}| \rightarrow 0$, both $E_{U D}(p)$ and $S_{U D}(p)$ in the numerator of the right-hand side of Eq. (5.17) will approach zeros. This means that at a low energy scale, $\phi^{-}$and $\phi^{+}$could still be considered as massless bound states and identified with charged Nambu-Goldstone bosons.

\section{CONCLUSIONS}

We have expounded electroweak symmetry breaking at finite temperature in a one-generation fermion condensate scheme in the real-time formalism of thermal field theory and in the fermion bubble approximation. It is proved by means of direct calculations of the propagators for bound states that, at the temperature $T$ below the symmetry restoration temperature $T_{c}$, it is always possible to obtain a massive neutral scalar bound state $\phi_{S}^{0}$, a massless neutral pseudoscalar bound state $\phi_{P}^{0}$, and two massless charged scalar bound states $\phi^{-}$and $\phi^{+}$if the two flavors of the one generation of fermions are mass degenerate. In this case, we can precisely identify $\phi_{S}^{0}$ with the Higgs boson and $\phi_{P}^{0}, \phi^{\mp}$ with the three Nambu-Goldstone bosons which appear as the products of the spontaneous breaking of electroweak group $\mathrm{SU}_{L}(2) \times \mathrm{U}_{Y}(1) \rightarrow \mathrm{U}_{Q}(1)$. In other words, the Goldstone theorem is valid rigorously in the fermion mass-degenerate case. On the other hand, when the two fermion flavors have unequal masses, we have seen that the Higgs boson will show double masses due to the effect of "thermal flactuation" except one of the two flavors being massless, and the two charged scalar bosons $\phi^{\mp}$ will also not be exactly massless. However, we find that as long as the momentum cutoff $\Lambda$ of the zero-temperature sectors of the fermion loops is sufficiently large or one is dealing with low energy scales of the bound states, then it is still possible approximately to obtain a single-Higgs-boson mass and almost massless $\phi^{\mp}$. In this case we can say that the Goldstone theorem is only valid approximately at a finite temperature. The well-known top-quark condensate scheme [8] certainely belongs to the latter case. Whether the appearence of such a situation originates from the real-time formalism itself of thermal field theory deserves to be examined further. Nevertheless, our discussions have shown that all possible pinch singularities cancel each other and do not emerge from the final expressions and this is just the result expected in a real-time thermal field theory. It is worth researching further if the above results based on the Schwinger-Dyson equation in the real-time formalism of thermal field theory could also appear in the imaginary-time formalism or in an effective potential approach.

\section{ACKNOWLEDGMENTS}

This work was done (in part) with the support of the Abdus Salam International Centre for Theoretical Physics, Trieste, Italy. It was also partially supported by the National Natural Science Foundation of China and by Grant No. LWTZ-1298 of the Chinese Academy of Sciences.

[1] D. A. Kirzhnits and A. D. Linde, Phys. Lett. 42B, 471 (1972); S. Weinberg, Phys. Rev. D 7, 2887 (1973); 9, 3357 (1974); L. Dolan and R. Jackiw, ibid. 9, 3320 (1974). 
[2] A. D. Linde, Rep. Prog. Phys. 42, 389 (1979); L. Girardello, M. T. Grisaru and P. Salomonson, Nucl. Phys. B178, 331 (1981); B. deWitt, in:Fundamental Interactions, Cargèse 1981, edited by. M. Levy et al. (Plenum, New York, 1982); R. H. Brandenberger, Rev. Mod. Phys. 57, 1 (1985).

[3] H. Umezawa, H. Matsumoto, and M. Tachiki, Thermo-field dynamics and condensed matter states (North-Holland, Amsterdam, 1982); Y. Fujimoto, R. Grigjanis and R. L. Kobes, Prog. Theor. Phys. 73, 434 (1985); I. Ojima, in Quantum Field Theory, edited by F. Mancini (North-Holland, Amsterdam, 1986); Y. Fujimoto and R. Grigjanis, Z. Phys. C 28, 395 (1985); Prog. Theor. Phys. 74, 1105 (1985); Y. Fujimoto and H. Nishino, Phys. Rev. D 32, 2167 (1985); A. J. Niemi and G. W. Semenoff, Nucl. Phys. B 230 [FS10], 181 (1984); D. Jonston, Z. Phys. C 31, 129 (1986).

[4] N. P. Landsman and Ch. G. van Weert, Phys. Rep. 145, 141 (1987), and the references therein.

[5] J. I. Kapusta, Finite-temperature field theory, (Cambridge University Press, Cambridge, England, 1989).

[6] B. Rosenstein, B. J. Warr, and S. H. Park, Phys. Rep. 205, 59 (1991) and references therein.

[7] Y. Nambu, Phys. Rev. Lett. 4, 380 (1960); J. Goldstone, Nuovo Cimento 19, 154 (1961); Y. Nambu and G. Jona-Lasinio, Phys. Rev. 122, 345 (1961); 124, 246 (1961); J. Goldstone, A. Salam and S. Weinberg, ibid. 127, 965 (1962); S. Bludman and A. Klein, ibid. 131, 2363 (1962).

[8] Y. Nambu, in New Theories in Physics, Proceedings of the XI International Symposium on Elementary Particle Physics, Kazimierz, Poland, 1988, edited by Z. Ajduk, S. Porkorski, and A. Trautman (World Scientific, Singapore, 1989); V. A. Miransky, M. Tanabashi and K. Yamawaki, Mod. Phys. Lett. A 4, 1043 (1989); Phys. Lett. B 221, 177 (1989). W. A. Bardeen, C. T. Hill, and M. Lindner, Phys. Rev. D 41, 1647 (1990).

[9] B. R. Zhou, Commun. Theor. Phys. 19, 337 (1993).

[10] B. R. Zhou, Phys. Rev. D 47, 5038 (1993).

[11] B. R. Zhou, Institution Report No AS-GS-TP-004, 1998, hep-th/9901 .

[12] B. R. Zhou, Commun. Theor. Phys., (to be published), hep-ph/9901 .

[13] B. R. Zhou, Phys. Rev. D 57, 3171 (1998). 